\title{
2D Video Analysis System to Analyze the Performance Model of Figure Roller Skating: A Pilot Study ${ }^{\dagger}$
}

\author{
Cristian Romagnoli 1,2,*, Vincenzo Bonaiuto ${ }^{2, *}$, Giorgio Gatta ${ }^{1}$, Naomi Romagnoli ${ }^{3}$, Anas \\ Alashram ${ }^{4}$, Elvira Padua ${ }^{5}$ and Giuseppe Annino ${ }^{6}$ \\ 1 Department for Life Quality Studies University of Bologna, I47037 Rimini, Italy; giorgio.gatta@unibo.it \\ 2 Sport Engineering Lab, Department Industrial Engineering, University of Rome Tor Vergata, 00133 Rome, \\ Italy \\ 3 Coach of the Italian Roller Sports Federation, Italy; naomiromagnoli@gmail.com \\ 4 School of Neuroscience, Faculty of Medicine and Surgery, University of Rome Tor Vergata, 00133 Rome, \\ Italy; anasalashram@gmail.com \\ 5 Department of Human Science and Promotion of Quality of Life, San Raffaele Open University of Rome, \\ 00166 Rome, Italy; elvira.padua@uniroma5.it \\ 6 Department of Medicine Systems, University of Rome, “Tor Vergata”, 00133 Rome, Italy; \\ g_annino@hotmail.com \\ * Correspondence: cristian.romagnoli2@unibo.it (C.R.); vincenzo.bonaiuto@uniroma2.it (V.B.) \\ + Presented at the 13th conference of the International Sports Engineering Association, Online, \\ 22-26 June 2020.
}

Published: 15 June 2020

\begin{abstract}
Figure roller skating is a discipline composed of various movements which involve jumps, artistic figures and spins in a seamless program which has both technical and shapely difficult. A biomechanical analysis of a double salchow was performed using a 2D video analysis of one European and in two Italian roller skaters. On average, the high level (HL) roller skater showed a horizontal velocity of the center of mass higher than the average, especially in the prop stage, whereas the medium level (ML) and low level (LL) athletes reduced their velocity significantly. The spin angular velocity of the ML and LL skaters was always higher than of the HL. This phenomenon would seem to be a compensatory strategy for a lower jump height, with a reduced trunk-thigh angle and less thigh lever arm (coxo-femur/knee joints) during the take-off and landing phases of the double salchow jump.
\end{abstract}

Keywords: video analysis; biomechanics; jumping performance; salchow; artistic figure roller skating

\section{Introduction}

In artistic roller skating, the explosive force parameters and explosive strength endurance represent the main performance limiting factors. Actually, the only studies present in the literature mainly concern artistic ice figure skating, which, for some aspects, can be used as a reference point in artistic roller figure skating. Podolsky et al., 1990, studied the relationship between muscle strength and jump height in ice figure skaters, and showed that the height of an axel and double axel is significantly correlated with the muscular strength of the shoulder in abduction and adduction, of the knee in extension and of the hip in extension and flexion, quantifying at $79.2 \%$ the influence of knee muscle extensors on height variability and at $5.5 \%$ the contribution of the shoulder muscles [1]. Some authors have argued that the activity of some muscle groups, such as the quadriceps, 
hamstrings and gastrocnemii, as well as the activity of the gluteus maximus, is fundamental for the success of skating jumping figures [2-4]. In fact, the electromyographic analysis of the lateral gastrocnemius muscles of the femoral biceps femoris, broad lateral and large gluteus had a greater activity during the execution of the jumps which included more spins (e.g., double axel and triple toe-loop) during both take-off and landing phases. For these jumps, especially the triple toe-loop, the biceps femoris appeared to be activated more frequently during the flight phase. In addition to the aforementioned characteristics, during the technical execution of the jumps, the athlete must also check other factors that contribute to the final performance model, such as the spin velocity of the body and the horizontal velocity at take-off and landing, which play a crucial role in the correct execution of the gesture, assuming a discriminating value in high-level skaters $[5,6]$. To date, no studies have investigated the biomechanical and neuromuscular aspect of artistic figure roller skating. The aims of the study are to evaluate and compare the performance model of the double salchow between high, medium and low technical level athletes.

\section{Materials and Method}

Three subjects of different technical levels participated in this study: a high level (HL) international roller skater (age: 18 yrs, height: $190 \mathrm{~cm}$, weight: $80 \mathrm{Kg}$ and $\mathrm{BMI}=22.1 \mathrm{Kg} / \mathrm{m}^{2}$ ), a medium level (ML) national roller skater (age: 16 yrs, height: $175 \mathrm{~cm}$, weight: $70 \mathrm{Kg}$, BMI: $22.85 \mathrm{Kg} / \mathrm{m}^{2}$ ) and a low level (LL) regional roller skater (age: 13 yrs, height: 150 cm, weight: $45 \mathrm{Kg}$, BMI: $20.00 \mathrm{Kg} / \mathrm{m}^{2}$ ). Written informed consent was obtained from all the participants after familiarization and an explanation of the benefits and risks involved in the procedures adopted. The study was approved by the University of Rome Ethical Committee "Tor Vergata". Moreover, all the tests were carried out in accordance with the Declaration of Helsinki.

To analyze the double salchow, 2D video analysis (BioMovie System by INFOLABMEDIA, Italy) was used. The video camera (CASIO EXILIM ZR 3700-CASIO CO.,LTD. Japan) was set at $240 \mathrm{fps}$ and placed at about $5 \mathrm{~m}$ from the center of the trajectory of where the jumps (double salchow) were performed [Figures 1 and 2]. A 2D directly linear transformation (2D-DLT) [7] was used for the calibration of the control object (vertical reference $100 \mathrm{~cm}$, horizontal reference $100 \mathrm{~cm}$ ), and to ensure that the skaters were in the same analysis plane. The horizontal axis was used for the calculation of the horizontal kinematic parameters. The shooting field, $7 \mathrm{~m}$ wide horizontally, was fractioned into 7 sub-units of $100 \mathrm{~cm}$ each. The time and horizontal displacement were measured to derive horizontal velocity $(\mathrm{m} / \mathrm{s})$, angular spin velocity $\left[1\right.$ spin $=360^{\circ} /$ time (or spin fraction) $\Rightarrow \% / \mathrm{s}$, thigh-trunk angle and thigh lever arm $(\mathrm{cm})$ (coxo-femur/knee joints). Jump height was calculated through the fly time (from take-off to landing) equation: $\boldsymbol{H}=\boldsymbol{T} \boldsymbol{f} * \frac{g}{8}[8]$.
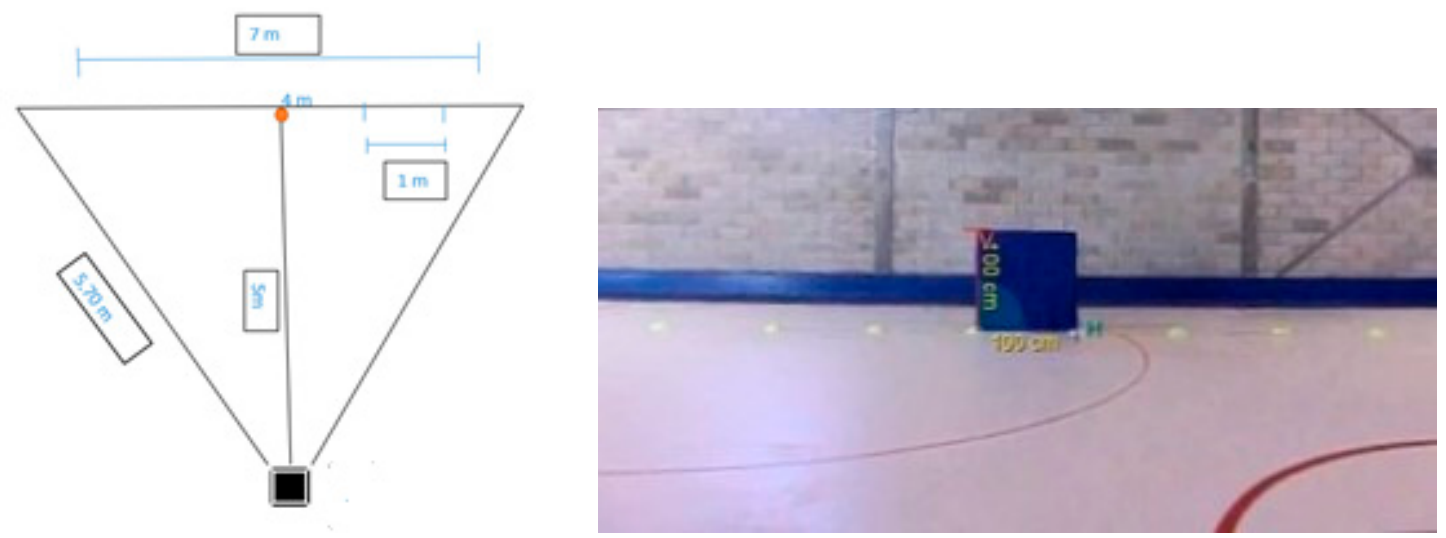

Figure 1. Shooting field. 


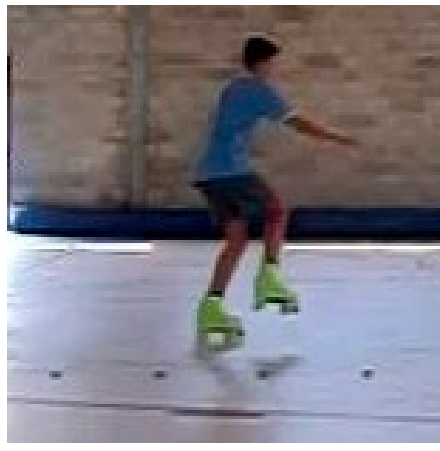

(a) Take-off phase

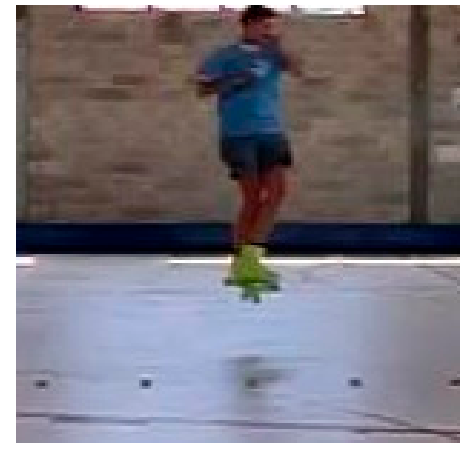

(b) Spin phase

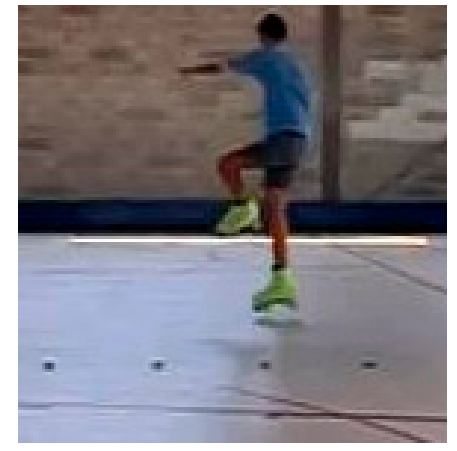

(c) Landing phase

Figure 2. Double Salchow sequences.

\section{Results}

In Figure 3, it is possible to observe the horizontal velocity, where the HL during the take-off performed at a higher velocity than the other athletes (green arrow). The increase that the roller skaters reached at 2-3 $\mathrm{m}$ of the horizontal displacement represents the lower and upper limbs' contribution before to the take-off.

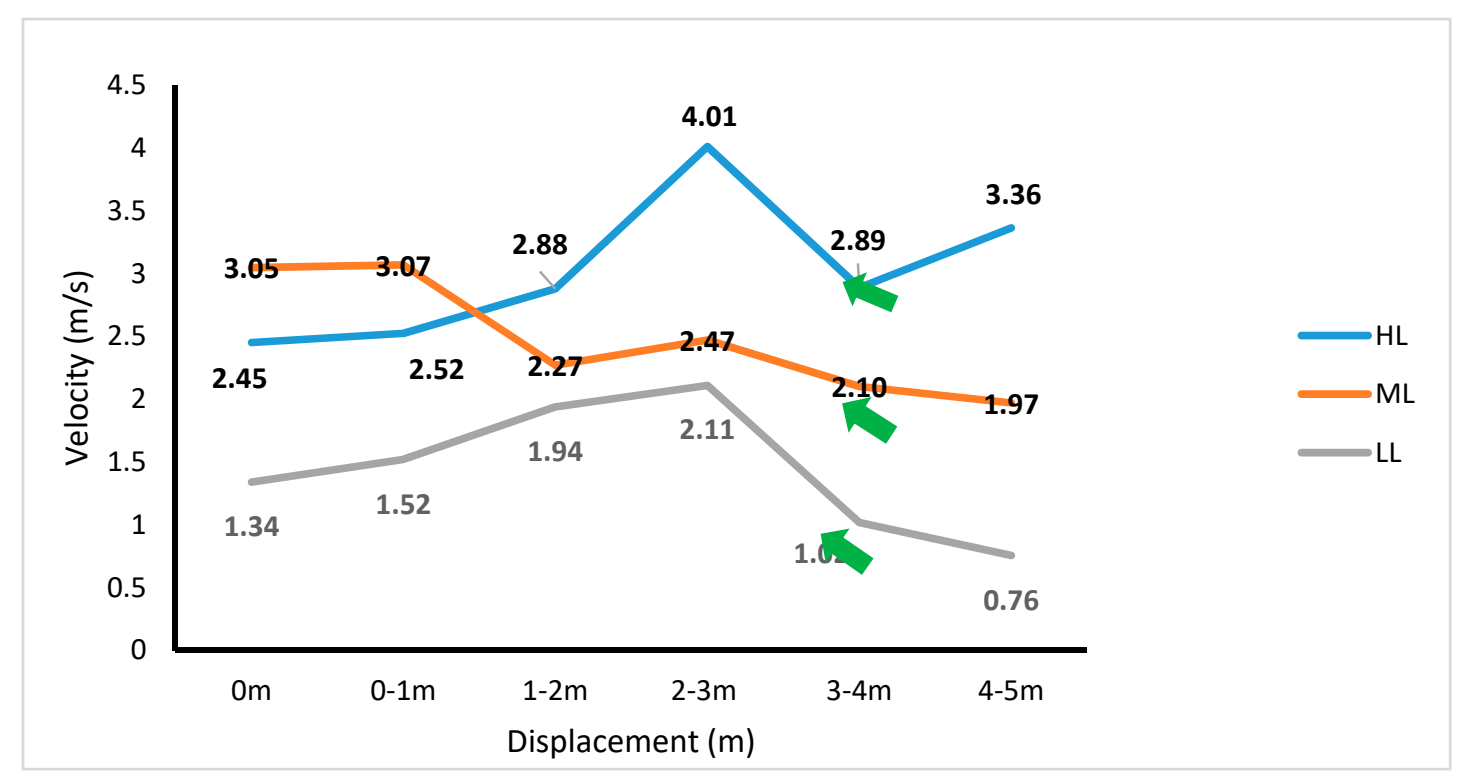

Figure 3. Horizontal velocity of each athlete, recorded during a double salchow jump.

Figure 4 shows the skater's strategy during the first $90^{\circ}$ and last $90^{\circ}$ of a jump's spin. In the first phase, the HL reached an angular spin velocity of $579.43^{\circ} / \mathrm{s}$, while in the final phase his velocity decreased at $566.34 \%$ s. Instead, the ML and LL show a higher angular spin velocity and a different strategy in both jumps' phase compared to the HL. In fact, the ML and LL's angular spin velocity at the landing show a higher velocity than the take-off phase. 


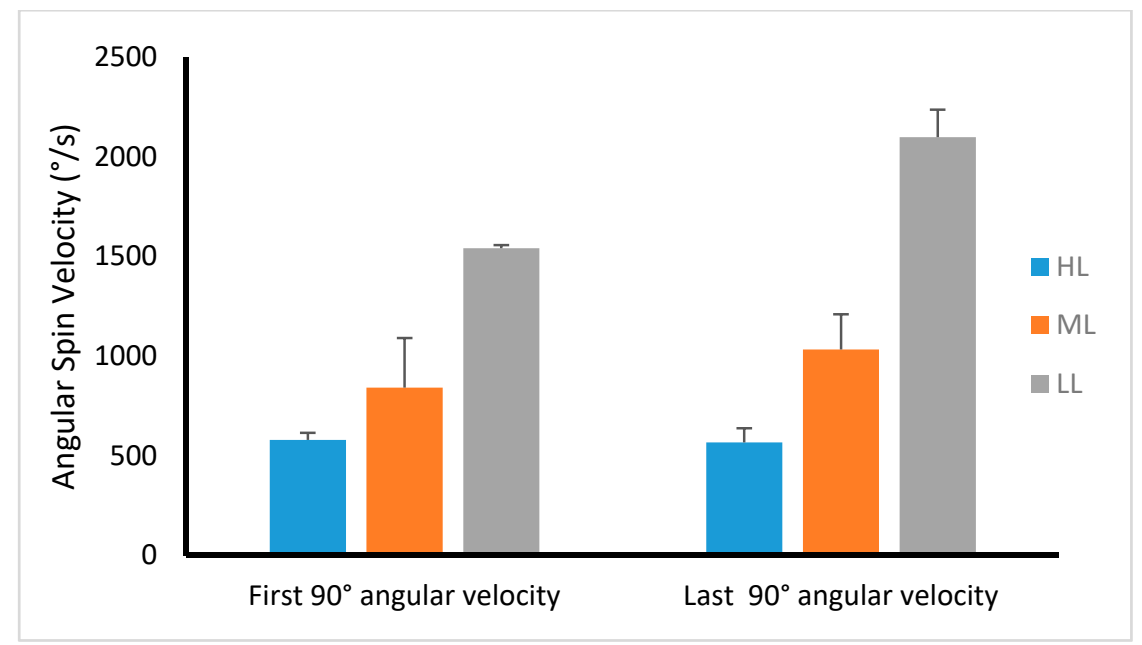

Figure 4. Angular spin velocity reached by each athlete during the double salchow performance at the take-off and landing phases.

Considering the thigh lever arm measurement (coxo-femur/knee joints) at the take-off and landing phases (Figure 5), the HL at the take-off had $47.85 \mathrm{~cm}$, while at the landing the HL increased his thigh lever arm at $50.80 \mathrm{~cm}$. Differently, the ML and LL adopted the opposite behavior, increasing their thigh lever arm at the take-off, and landing with a reduced thigh lever arm.

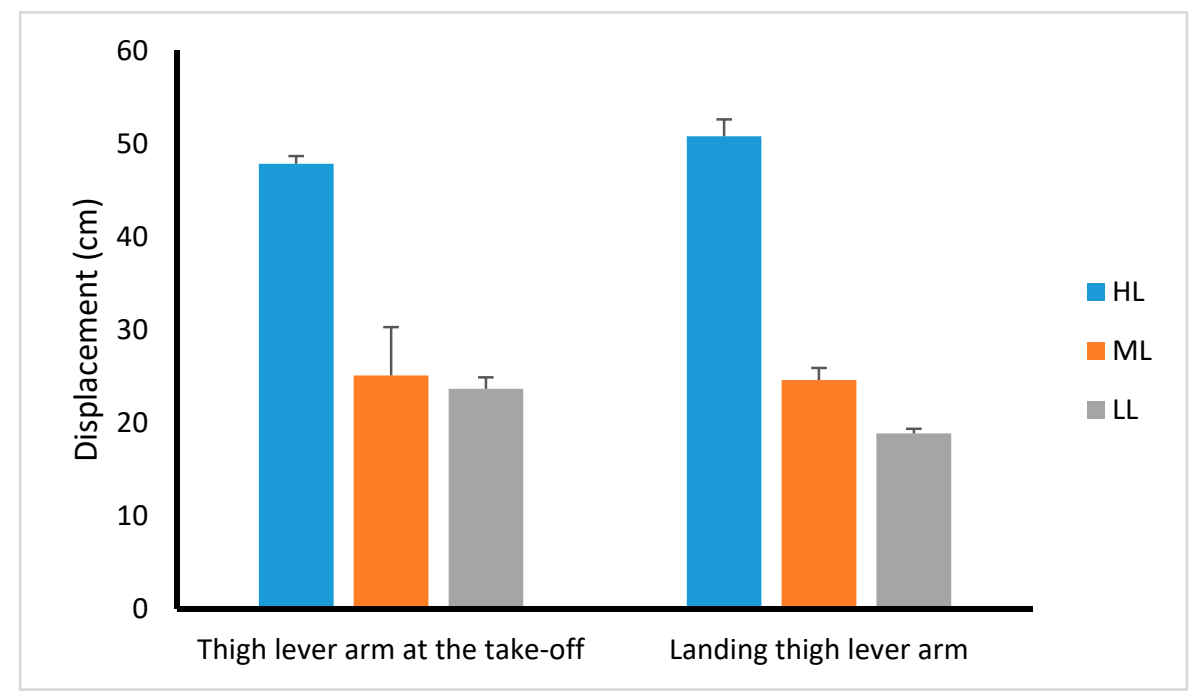

Figure 5. Thigh lever arm (coxo-femoral/knee joints) during the take-off and the landing of the double salchow.

The consequence of the two different strategies observed for thigh lever arm measure is related to the thigh-trunk angle at the take-off and landing phases. In fact, as shown in Figure 6, the HL at the take-off had a thigh-trunk angle lower than in the landing phase, while the ML and LL had a greater angle at the take-off than in the landing phase. 


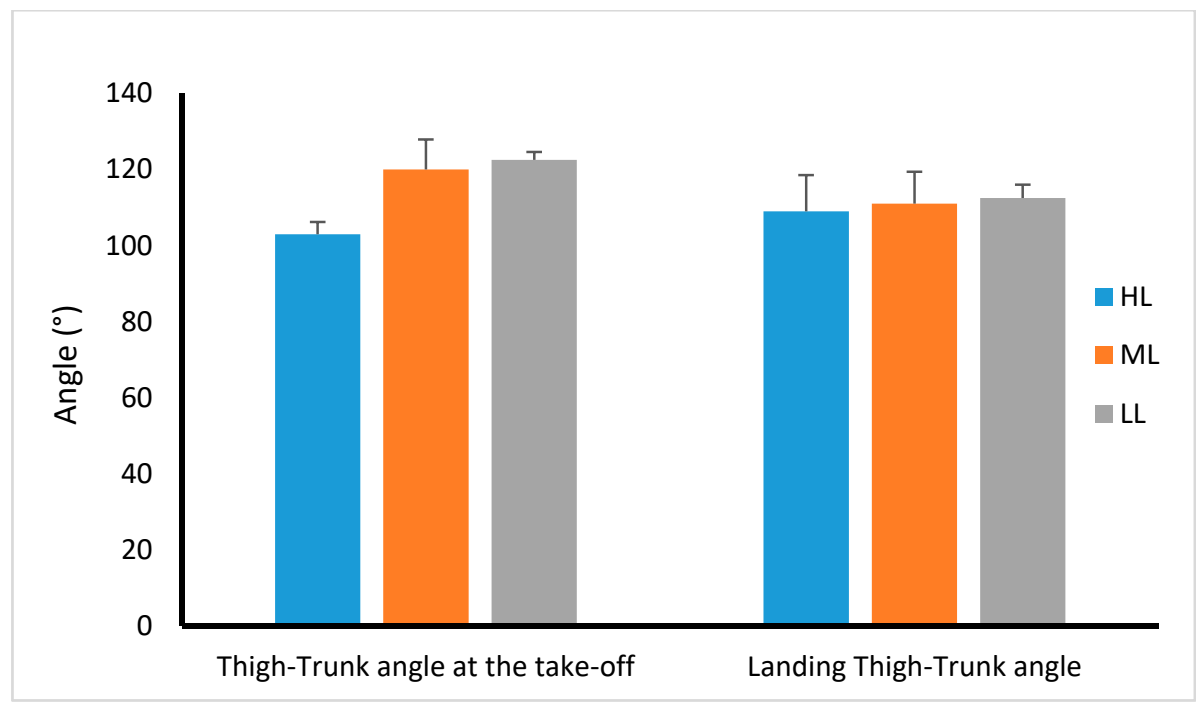

Figure 6. Thigh-trunk angle at the take-off and landing phases during the double salchow performance of each athlete.

Figure 7 represents the vertical height reached during the double salchow for each athlete. The HL skater showed a greater vertical height $(29.92 \mathrm{~cm})$ than the ML $(21.81 \mathrm{~cm})$ and LL $(12.21 \mathrm{~cm})$.

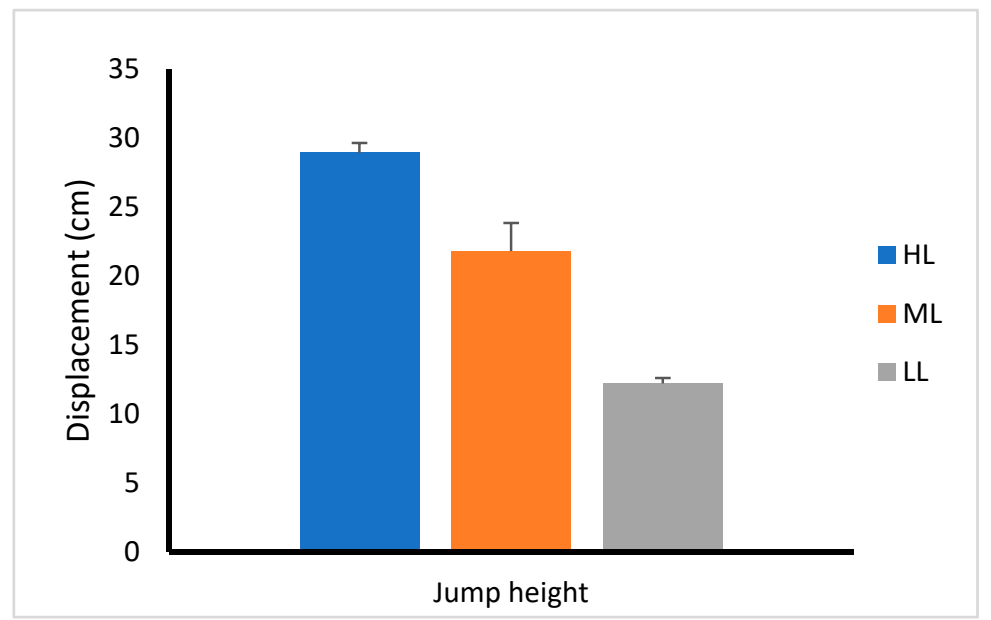

Figure 7. Vertical height of double salchow reached by HL, ML, LL.

\section{Discussion}

All the parameters analyzed in this study could be considered as discriminating factors between HL, ML and LL in figure roller skating. Considering the horizontal velocity, the HL in the preparatory phase of the jump showed a higher velocity than the ML and LL, which tended to decrease in the following landing phases, while the ML and LL always had lower velocity in all the jump phases analyzed (Figure 3). This difference is probably due to the greater technical management ability of the HL, who was also able to perform triple salchow jumps, with respect to the ML and LL. This ability is also related to higher fly time, as shown in the double salchow jump (Figure 7), and in the better combination of angular spin velocity shown at the take-off and landing phases (Figure 4). In fact, the HL strategy showed a less angular spin velocity at take-off and in landing phases, managed through a greater length thigh lever arm (Figure 5) and lower thigh-trunk angle (Figure 6), with respect the ML and LL. In addition, the HL's strategy resulted in a higher fly time and vertical jump height reached during the double salchow performance. For this reason, it seems that explosive muscle extensor strength could be considered a limiting factor of artistic figure roller skating, as shown previously by Podolsky et al.'s 1990 observation of artistic figure ice skating. This result represents a study of, primarily, the biomechanical and neuromuscular aspects of the artistic figure 
roller skating discipline. Obviously, a future study with a greater sample size will be needed to confirm these preliminary results.

\section{Conclusions}

From this pilot study, where analyses were only performed on three athletes of different levels, enough information emerged to give a clearer picture of the technical and biomechanical parameters which contribute to the performance model; the data obtained had discriminating features between levels in terms of horizontal velocity, angular spin velocity and jump height. Obviously, the management of higher horizontal velocity and jump height are related to higher explosive strength of the leg muscle extensor. Moreover, 2D video analysis is an important tool for the evaluation of the technique or technical elements which characterize the performance model in artistic roller skating.

Funding: This research received no external funding

Acknowledgments: The authors would like to thank Naomi Romagnoli and Federico Tassini for technical support during the jump's analysis, and the Talent's A.S.D for the availability of her athletes.

Conflicts of Interest: The authors declare no conflict of interest.

\section{References}

1. Podolsky, A.; Kaufman, K.R.; Cahalan, T.D.; Aleshinsky, S.Y.; Chao, E.Y. The relationship of strength and jump height in figure skaters. Am. J. Sports Med. 1990, 18, 400-405.

2. Aleshinsky, S.Y.; Smith, S.L.; Jansen, L.B.; Ramirez, E.F. Comparison of biomechanical parameters demonstrated by Brian Boitano in Triple and Double Axel jumps. J. Biomech. 1988, 21, 882.

3. King, D.L. Performing triple and quadruple figure skating jumps: Implications for training. Can. J. Appl. Physiol. 2005, 30, 743-753.

4. Poe, C.M.; O'Bryant, H.S.; Laws, D.E. Off-ice resistance and plyometric training for singles figure skaters. Strength Condit. J. 1994, 16, 68-76.

5. King, D.L.; Arnold, A.S.; Smith, E.S.L. A kinematic comparison of single, double, and triple axels. J. Appl. Biomech. 1994, 10, 51-60.

6. King, D.; Smith, S.; Higginson, B.; Muncasy, B.; Scheirman, E.G. Figure Skating: Characteristics of triple and quadruple toe-loops performed during the Salt Lake City 2002 winter Olympics. Sports Biomech. 2004, 3, 109-123.

7. Brewin, M.A.; Kerwin, D.G. Accuracy of scaling and DLT reconstruction techniques for planar motion analyses. J. Appl. Biomech. 2003, 19, 79-88.

8. Asmussen, E.; Bonde-Petersen, F. Storage of elastic energy in skeletal muscles in man. Acta Physiol. Scand. 1974, 91, 385-392.

(C) 2020 by the authors. Licensee MDPI, Basel, Switzerland. This article is an open access article distributed under the terms and conditions of the Creative Commons Attribution (CC BY) license (http://creativecommons.org/licenses/by/4.0/). 